\title{
Optimization of an In Silico Cardiac Cell Model for Proarrhythmia Risk Assessment
}

\author{
Sara Dutta ${ }^{1}$, David Strauss ${ }^{1}$, Thomas Colatsky ${ }^{2}$, Zhihua $\mathrm{Li}^{1}$ \\ ${ }^{1}$ U.S. Food and Drug Administration, Silver Spring, MD, USA; ${ }^{2}$ Seabrook Island SC, USA
}

\begin{abstract}
The Comprehensive in vitro Proarrhythmia Assay (CiPA) is a regulatory paradigm proposed to replace the ICH S7B and E14 guidelines for assessing drug-induced proarrhythmia. Under CiPA, drug effects on multiple cardiac ion channels will be measured in vitro and integrated into an in silico model of the adult human ventricular cell, based on the O'Hara-Rudy (ORd) model. However, the ORd model does not accurately represent certain ionic currents known to be critical in triggering drug-induced arrhythmias, such as the late sodium current $\left(I_{N a L}\right)$. The goal of the present study is to systematically assess and improve the simulation of the main depolarizing and repolarizing ionic currents (the inward rectifying potassium currents, L-type calcium current and $I_{N a L}$ ) in the ORd model. We present a new model with scaled conductances calculated by fitting to O'Hara et al. in vitro human cardiomyocyte channel blocking experiments using a genetic algorithm, which improves discrepancies of the original model. The modified model particularly improves the effect of $I_{N a L}$ block on action potential prolongation, an important determinant of proarrhythmia risk in the context of CiPA.
\end{abstract}

\section{Introduction}

Torsade-de-pointes (TdP) is a lethal type of arrhythmia that caused removal of several drugs from the market [1] and led to the adoption of the ICH E14 and S7B guidelines to identify TdP risk. Although block of the delayed rectifier potassium current $\left(\mathrm{I}_{\mathrm{Kr}}\right)$ and the $10 \mathrm{~ms}$ prolongation of QT criteria set by the guidelines are highly sensitive predictors of TdP risk, they are not specific and may prevent many useful and effective drugs with low TdP risk from entering the market. In an effort to improve the specificity of assessing clinical TdP risk, the Comprehensive in vitro Proarrhythmia Assay (CiPA) collaborative initiative was established [2]. The aim of this regulatory paradigm is to combine measurements of multiple cardiac ionic currents with in silico modeling to predict TdP risk, coupled with stem cell and clinical ECG studies to confirm those predictions or identify mechanisms missing from the patch clamp data sets. The O'Hara et al. model (ORd) was chosen as the consensus base model [3].

As part of CiPA, an initial set of 12 drugs determined by cardiologists to have low, intermediate or high risk of TdP were selected to form a training set. Many of the CiPA drugs are multi-channel blockers, particularly the low risk drugs [4]-[6]. However, simulations of drug effects using the current version of the ORd model and latest patch clamp data show discrepancies with results on human cardiomyocytes, suggesting some currents (eg. the late sodium current, $\mathrm{I}_{\mathrm{NaL}}$ ) are not correctly represented.

The aim of this study is to improve the ORd model so that it accurately simulates the changes in action potential duration (APD) produced by drugs that block multiple ion channels.

\section{Methods}

\subsection{Simulation protocol}

All simulations were run using the ORd endocardial cell model for 1000 beats at varying cycle lengths (CLs) 500, 1000, 2000 and 4000 ms. Drug block was simulated using the Hill function and data from Crumb et al. [7] for varying drug concentrations: free plasma clinical drug exposures (Cmax) up to 20X Cmax. APD was calculated as the time taken for the transmembrane potential $\left(\mathrm{V}_{\mathrm{m}}\right)$ of the cell to reach 90\% (APD90), 70\% (APD70), 50\% (APD50) and 30\% (APD30) of its resting $\mathrm{V}_{\mathrm{m}}$. $\triangle \mathrm{APD}$ was calculated as the percentage difference in APD prolongation between control and drug. Simulations were run in $\mathrm{R}$ and $\mathrm{C}$ using the deSolve package.

\subsection{Modified ORd model}

The original ORd model was modified by scaling conductances as follows: $\mathrm{I}_{\mathrm{Kr}}$ by 1.119 , the slow rectifier potassium current $\left(\mathrm{I}_{\mathrm{Ks}}\right)$ by 1.648 , the inwardly rectifying potassium current $\left(\mathrm{I}_{\mathrm{K} 1}\right)$ by 1.414 , the L-type calcium current $\left(\mathrm{I}_{\mathrm{CaL}}\right.$ ) by 1.018 and $\mathrm{I}_{\mathrm{NaL}}$ by 2.274 . These values were calculated by fitting to experimental data from 
O'Hara et al. using a Genetic Algorithm-based Parameterization for Systems Modeling [8]. Briefly, an initial set of parameters are defined within a certain range and their goodness of fit is assessed using an objective function defined as the weighted sum of the squared errors between model values and experimental measurements. The set of parameters then undergoes various changes (i.e. mutation and recombination) to create a new generation of parameters and this process is continued until a global optimum is reached.

The experimental data used in the algorithm are taken from the ORd model paper [3] and shows APD rate dependence for control and 5 drug blocking conditions: 1 $\mu \mathrm{M}$ E-4031 (70\% I $\mathrm{I}_{\mathrm{Kr}}$ block), $1 \mu \mathrm{M}$ HMR-1556 (90\% I $\mathrm{I}_{\mathrm{Ks}}$ block), $1 \mu \mathrm{M}$ nisoldipine (90\% $\mathrm{I}_{\mathrm{CaL}}$ block), $100 \mu \mathrm{M}$ BaCl2 (90\% $\mathrm{I}_{\mathrm{K} 1}$ block), $10 \mu \mathrm{M}$ mexiletine (54\% $\mathrm{I}_{\mathrm{NaL}}, 9 \%$ $\mathrm{I}_{\mathrm{Kr}}$, and $20 \% \mathrm{I}_{\mathrm{CaL}}$ block). The simulated block was kept the same as in the ORd paper, apart from mexiletine, which was simulated using IC50 and hill coefficient data from Crumb et al. [8]. The algorithm was run using inhouse developed $\mathrm{R}$ scripts and parallel computing Snow and Rmpi packages on the FDA High Performance Computer (HPC) with 160 cores.

\section{Results}

\subsection{Fitting to experimental data}

The modified ORd model was built by refitting to APD rate dependence experimental data from O'Hara et al., as shown in Figure 1 and Table 1. The goodness of fit of each set of parameters generated by the genetic algorithm was tested by calculating the sum of squares error as in Table 1. The best parameters were passed onto the next generation and the rest underwent mutation, recombination, migration and repopulation to create the new set of parameters. Here we present the best parameters that showed the smallest error.

Under control conditions both the original and modified models display a similar behavior, although APD90 is shorter in the modified ORd. However, the overall error under control conditions is smaller for the modified ORd (15.01) than the original ORd (17.20) models (see Table 1). Furthermore, the modified ORd model shows a better fit to the experimental data for the $\mathrm{I}_{\mathrm{NaL}}, \mathrm{I}_{\mathrm{CaL}}$ and $\mathrm{I}_{\mathrm{Kr}}$ blockers (23.98 vs. 92.92; 0.75 vs. 5.29; 145.03 vs. 15.96) while the two models show similar results for the $\mathrm{I}_{\mathrm{Ks}}$ blocker (HMR-1556). Finally, the modified ORd shows good agreement with experimental data for faster CLs $\leq 1000 \mathrm{~ms}$ for the $\mathrm{I}_{\mathrm{K} 1}$ blocker $\left(\mathrm{BaCl}_{2}\right)$. Therefore, overall the modified ORd displays a closer match to experimental data (average error of 19.85 vs. 57.77). The main improvements are observed for the $\mathrm{I}_{\mathrm{NaL}}$ and $\mathrm{I}_{\mathrm{Kr}}$ blockers, mexiletine and E-4031.

\subsection{AP and current traces of the models}

As described in the methods all of the current conductances are increased in the modified ORd model, however, the AP shape of both models is very similar, as shown in Figure 2. The smallest change in current amplitude observed is $\mathrm{I}_{\mathrm{Ca}}$, which only has a $1.8 \%$ change in conductance. However, clear differences are observed for all other currents ( $\mathrm{I}_{\mathrm{Kr}}, \mathrm{I}_{\mathrm{NaL}}, \mathrm{I}_{\mathrm{Ks}}$ and $\mathrm{I}_{\mathrm{K} 1}$ ) with the biggest changes occurring for $\mathrm{I}_{\mathrm{NaL}}$ (conductance is increased by 227\%) and $\mathrm{I}_{\mathrm{Ks}}$ (conductance is increased by $165 \%)$. Therefore, $\mathrm{I}_{\mathrm{NaL}}$ plays a bigger role in the modified ORd model given there is a greater increase in $\mathrm{I}_{\mathrm{NaL}}$ compared to $\mathrm{I}_{\mathrm{Kr}}$.
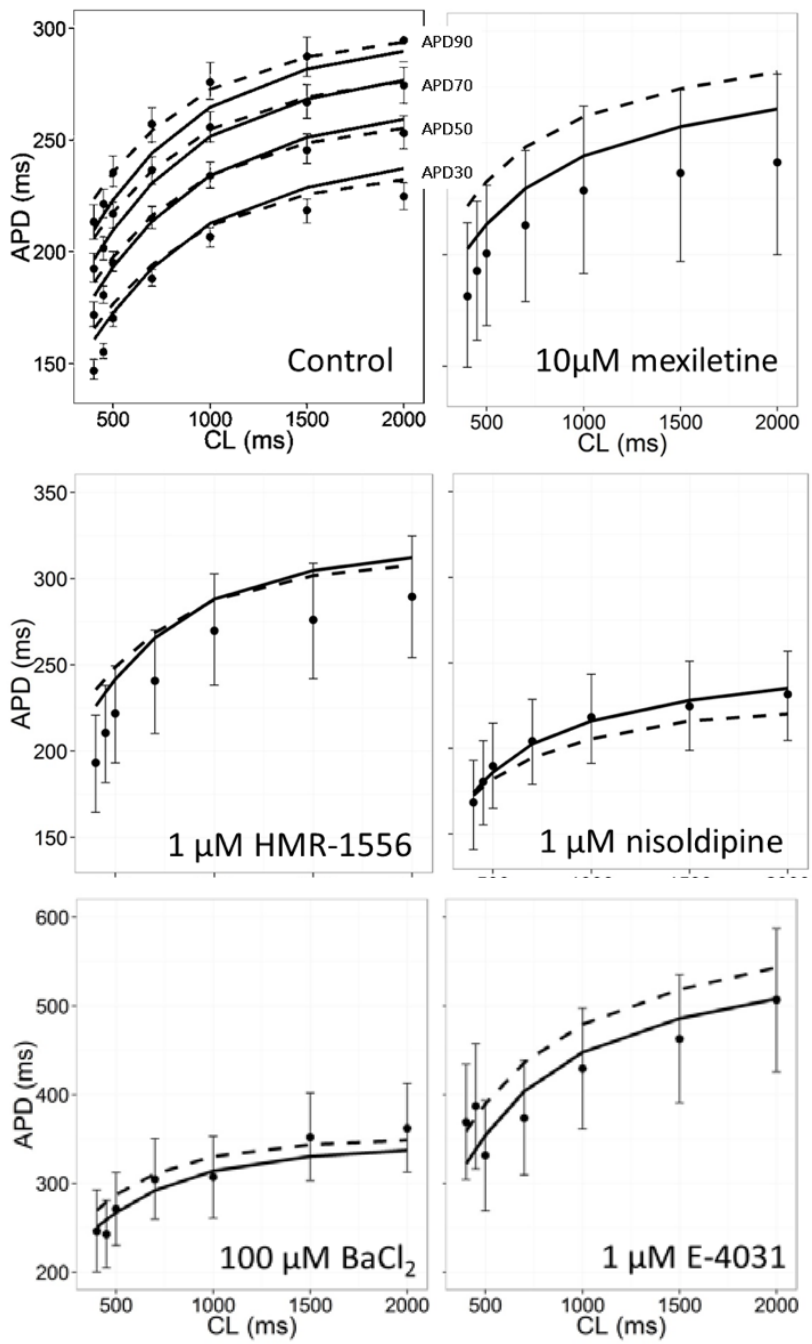

Figure 1. Control, $10 \mu \mathrm{M}$ mexiletine, $1 \mu \mathrm{M}$ HMR-1556, 1 $\mu \mathrm{M}$ nisoldipine, $100 \mu \mathrm{M} \mathrm{BACl}_{2}$ and $1 \mu \mathrm{M}$ E-4031 steady state APD rate dependence for varying cycle lengths (CLs) for the original O'Hara et al. (ORd; dashed lines), the modified ORd model (solid line) and experimental data mean and standard deviation from O'Hara et al. [3] (error bars). Control shows action potential duration 
(APD) at 90\% (APD90), 70\% (APD70), 50\% (APD50) and $30 \%$ (APD30) repolarization. All other panels show APD90.

Table 1. Sum of squares error between APD rate dependence experimental data mean and simulation results (see Figure 1) for the original ORd and the modified ORd.

\begin{tabular}{lll}
\hline Sum of squares error & Original ORd & $\begin{array}{l}\text { Modified } \\
\text { ORd }\end{array}$ \\
\hline Control & 17.2 & 15.01 \\
$10 \mu \mathrm{M}$ mexiletine & 92.92 & 23.98 \\
$1 \mu \mathrm{M} \mathrm{HMR}-1556$ & 56.35 & 43.54 \\
$1 \mu \mathrm{M} \mathrm{E}-4031$ & 145.03 & 15.96 \\
$100 \mu \mathrm{M} \mathrm{BaCl}$ & 29.83 & 15.96 \\
$1 \mu \mathrm{M} \mathrm{nisoldipine}$ & 5.29 & 0.75 \\
Average \pm SD & $\mathbf{5 7 . 7 7} \pm \mathbf{5 2 . 9 8}$ & $\mathbf{1 9 . 8 5} \pm \mathbf{1 6 . 6 7}$ \\
\hline
\end{tabular}
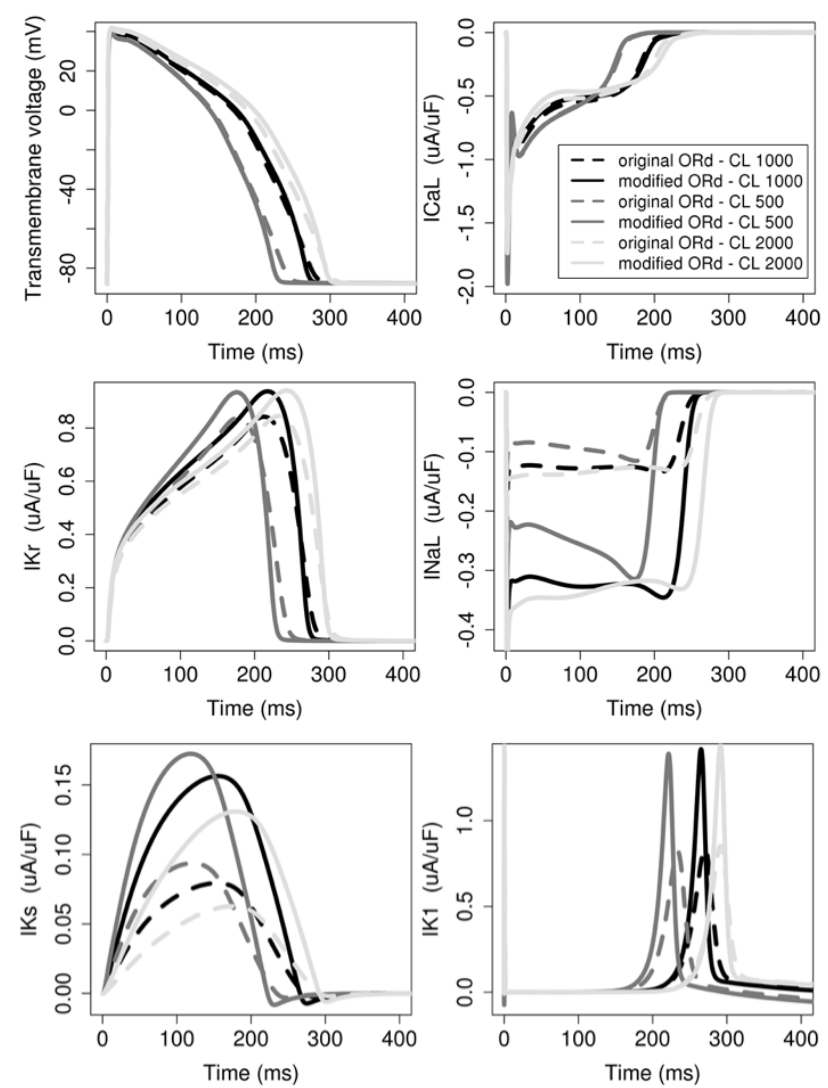

Figure 2. Action potential (AP), L-type calcium current ( $\left.\mathrm{I}_{\mathrm{CaL}}\right)$, delayed rectifier potassium current $\left(\mathrm{I}_{\mathrm{Kr}}\right)$, late sodium current $\left(\mathrm{I}_{\mathrm{NaL}}\right)$, slow rectifier potassium current ( $\left.\mathrm{I}_{\mathrm{Ks}}\right)$ and inwardly rectifying potassium current $\left(\mathrm{I}_{\mathrm{K} 1}\right)$ traces under control conditions for the original ORd (dashed line) and the modified ORd (solid line) for CLs of 500 (dark gray), 1000 (black) and 2000 (light gray) ms.

\subsection{Drug-induced APD prolongation}

Block of $I_{\text {NaL }}$ is underestimated and block of $I_{K r}$ is overestimated in the original ORd model, as shown in Figure 3. The modified ORd shows a smaller APD prolongation for $75 \% \mathrm{I}_{\mathrm{Kr}}$ block compared to the original ORd (192.5 ms vs $238.52 \mathrm{~ms}$ ) and a greater decrease in APD for $75 \% \mathrm{I}_{\mathrm{NaL}}$ block (42.51 ms vs. $16.5 \mathrm{~ms}$ ). As shown in Figure 1, this is closer to the experimental data with E-4031, a potent $\mathrm{I}_{\mathrm{Kr}}$ blocker, and mexiletine, a potent $\mathrm{I}_{\mathrm{NaL}}$ blocker. Proper characterization of $\mathrm{I}_{\mathrm{NaL}}$ block in the model is important as it plays an important role in counteracting pro-arrhythmic APD prolongation of $\mathrm{I}_{\mathrm{Kr}}$ block.

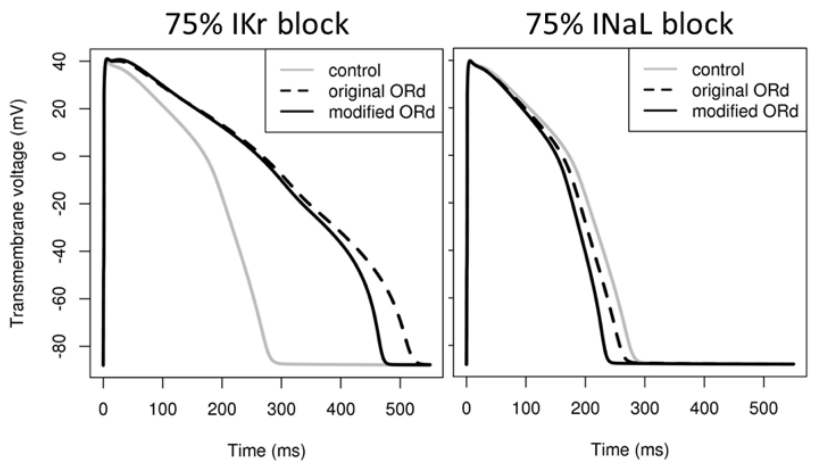

Figure 3. AP traces for $75 \% \mathrm{I}_{\mathrm{Kr}}$ (left panel) and $\mathrm{I}_{\mathrm{NaL}}$ block (right panel); original ORd (dashed black line); modified ORd (solid black line); control (gray).

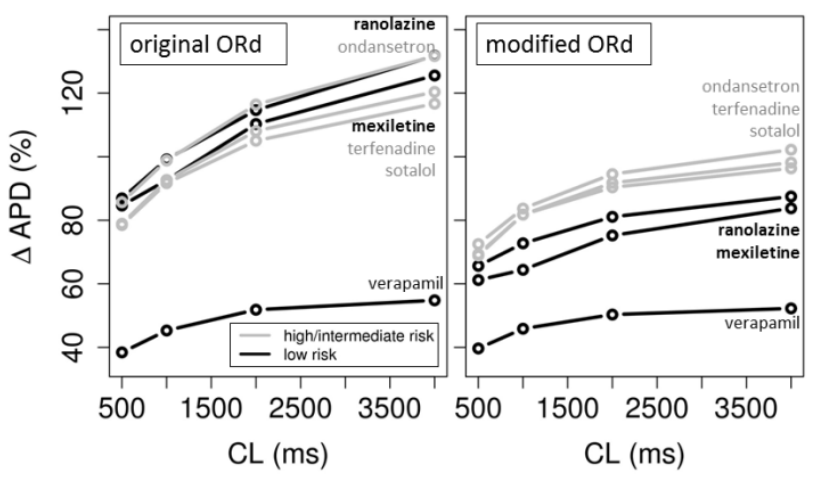

Figure 4. Original ORd (left panel) and modified ORd (right panel) $\triangle \mathrm{APD}(\%)$ with a CL of $500,1000,2000$ and $4000 \mathrm{~ms}$ and 20X Cmax for a subset of the CiPA drugs (high/intermediate risk in gray and low risk in black).

The increased effect of $\mathrm{I}_{\mathrm{NaL}}$ is demonstrated in Figure 4, which shows APDs for varying CLs in both models for a subset of the CiPA drugs. As expected for all models the APD prolongation increases as the CL increases. However, in the original ORd model ranolazine and mexiletine ( $\mathrm{I}_{\mathrm{NaL}}$ blockers) show an increase in APD similar to other high and intermediate risk drugs. This is improved in the modified ORd model, where the APD 
prolongation of the $\mathrm{I}_{\mathrm{NaL}}$ blockers is closer to other safe drugs such as verapamil ( $\mathrm{I}_{\mathrm{CaL}}$ blocker) and is clearly separated from higher risk drugs. Therefore, the separation between the safe risk category and the intermediate and high risk categories is improved in the modified ORd model.

\section{Discussion}

This study presents a modified ORd model with a new set of scaling conductances for the $\mathrm{I}_{\mathrm{Kr}}, \mathrm{I}_{\mathrm{NaL}}, \mathrm{I}_{\mathrm{CaL}}, \mathrm{I}_{\mathrm{Ks}}$ and $\mathrm{I}_{\mathrm{K} 1}$ currents that better fit APD rate dependence experimental data, improve the prediction of $\mathrm{I}_{\mathrm{NaL}}$ block on APD and the separation of the low TdP risk CiPA drugs from the intermediate and high risk CiPA drugs.

This study has certain limitations that should be taken into account. Firstly, IC50 current blocking data for high Cmax were extrapolated in some cases as the concentrations simulated were higher than the experimental concentrations tested by Crumb et al., as was the case for the $\mathrm{I}_{\mathrm{Kr}}$ blocking effect of mexiletine [7]. Secondly, APD, like QT, is not a predictive marker of drug-induced TdP risk [2]; therefore this metric is presented to show the change in APD prolongation induced by $\mathrm{I}_{\mathrm{CaL}}$ and $\mathrm{I}_{\mathrm{NaL}}$ block rather than attempting to show clear separation between the TdP risk categories. The authors of this study are investigating other more specific and sensitive metrics of TdP risk. Thirdly, dynamics of the $\mathrm{I}_{\mathrm{Kr}}$ current play an important role in the risk of TdP, particularly for high and intermediate risk drugs [9], [10], and therefore, an updated ORd model is currently being developed based on our previously published model [11]. Finally as shown in Figure 1, despite the change in $\mathrm{I}_{\mathrm{Ks}}$, the original and modified ORd models show similar results due to changes in $\mathrm{I}_{\mathrm{Ks}}$ having little effect on APD, as shown previously [12], however under certain conditions, such as beta-stimulation, effects may be more pronounced.

In conclusion, this study presents important results for the development of a modified ORd human ventricular action potential model that can more accurately predict drug-induced TdP, particularly in the presence of $\mathrm{I}_{\mathrm{NaL}}$ block.

\section{Acknowledgements}

We would like to thank Drs Jose Vicente, Robbert Zusterzeel, Wendy Wu, Jiansong Sheng, Phu N. Tran and Min Wu for their support and numerous discussions.

\section{References}

[1] G. A. Gintant, "Preclinical Torsades-de-Pointes Screens: Advantages and limitations of surrogate and direct approaches in evaluating proarrhythmic risk,” Pharmacol. Ther., vol. 119, no. 2, pp. 199-209, Aug. 2008.
[2] P. T. Sager, G. Gintant, J. R. Turner, S. Pettit, and N. Stockbridge, "Rechanneling the cardiac proarrhythmia safety paradigm: a meeting report from the Cardiac Safety Research Consortium.," Am. Heart J., vol. 167, no. 3, pp. 292-300, Mar. 2014.

[3] T. O’Hara, L. Virág, A. Varró, and Y. Rudy, “Simulation of the undiseased human cardiac ventricular action potential: model formulation and experimental validation.," PLoS Comput. Biol., vol. 7, no. 5, p. e1002061+, May 2011.

[4] M. R. Rosen, J. P. Ilvento, H. Gelband, and C. Merker, "Effects of verapamil on electrophysiologic properties of canine cardiac purkinje fibers,” J. Pharmacol. Exp. Ther., vol. 189, no. 2, pp. 414-422, May 1974.

[5] C. Antzelevitch, L. Belardinelli, A. C. Zygmunt, A. Burashnikov, J. M. Di Diego, J. M. Fish, J. M. Cordeiro, and G. Thomas, "Electrophysiological Effects of Ranolazine, a Novel Antianginal Agent With Antiarrhythmic Properties," Circulation, vol. 110, no. 8, pp. 904-910, Aug. 2004.

[6] W. Shimizu and C. Antzelevitch, "Sodium Channel Block With Mexiletine Is Effective in Reducing Dispersion of Repolarization and Preventing Torsade de Pointes in LQT2 and LQT3 Models of the Long-QT Syndrome," Circulation, vol. 96, no. 6, pp. 2038-2047, Sep. 1997.

[7] W. J. Crumb, J. Vicente, L. Johannesen, and D. G. Strauss, "An evaluation of 30 clinical drugs against the comprehensive in vitro proarrhythmia assay (CiPA) proposed ion channel panel," J. Pharmacol. Toxicol. Methods, Apr. 2016.

[8] Z. Li, H. Zhou, Y. Lu, and T. Colatsky, “A Critical Role for Immune System Response in Mediating Anti-influenza Drug Synergies Assessed by Mechanistic Modeling," CPT Pharmacomet. Syst. Pharmacol., vol. 3, p. e135+, 2014.

[9] J. T. Milnes, H. J. Witchel, J. L. Leaney, D. J. Leishman, and J. C. Hancox, "Investigating dynamic protocoldependence of hERG potassium channel inhibition at $37^{\circ} \mathrm{C}$ : Cisapride versus dofetilide,” J. Pharmacol. Toxicol. Methods, vol. 61, no. 2, pp. 178-191, Mar. 2010.

[10] G. Y. DI Veroli, M. R. Davies, H. Zhang, N. Abi-Gerges, and M. R. Boyett, "hERG inhibitors with similar potency but different binding kinetics do not pose the same proarrhythmic risk: implications for drug safety assessment.," J. Cardiovasc. Electrophysiol., vol. 25, no. 2, pp. 197-207, Feb. 2014.

[11] Z. Li, S. Dutta, J. Sheng, P. N. Tran, W. Wu, and T. Colatsky, "A temperature-dependent in silico model of the human ether-à-go-go-related (hERG) gene channel,” J. Pharmacol. Toxicol. Methods, May 2016.

[12] G. R. Mirams, M. R. Davies, S. J. Brough, M. H. Bridgland-Taylor, Y. Cui, D. J. Gavaghan, and N. AbiGerges, "Prediction of Thorough QT study results using action potential simulations based on ion channel screens.," J. Pharmacol. Toxicol. Methods, vol. 70, no. 3, pp. 246-254, 2014.

Address for correspondence.

Sara Dutta

U.S. Food and Drug Administration, Building 64, Room 2084, 10903 New Hampshire Ave, Silver Spring, MD 20993.

sara.dutta@fda.hhs.gov. 